\begin{tabular}{|l|l|}
\hline Asian Journal of Pharmaceutical Research & A \\
and Development & J \\
(And International Peer-Reviewed Journal of Pharmaceutical Research and Development) \\
@ 2013-18, publisher and licensee APRD, This is an Open Access article which permits unrestricted \\
non-commercial use, provided the original work is properly cited
\end{tabular}

Open $\odot$ Access

Available online at www.ajprd.com

Review Article

\title{
AN EXTENDED REVIEW AND SUMMARY ON FACTS ABOUT ETIOLOGY EPIDEMIOLOGY CLINICAL DIAGNOSIS AND MANAGEMENT OF SCRUB TYPHUS INFECTION G.V.Nagaraju, G.V.Pavan Kumar, Naga Subramanyam S.
}

\author{
Department of Pharmacy Practice, Koringa College of Pharmacy, Kakinada, A.P, India.
}

\begin{abstract}
Scrub typhus is also known as bus typhus inaction and it is a serious public health problem in the Asia-Pacific area. It threatens one billion peoples are effected worldwide, and causes illness in one million people for an each year. Caused by Orientia tsutsugamushi, scrub typhus can result in severe multi organ failure with a case fatality rate up to $70 \%$ without appropriate treatment. The antigenic heterogeneity of $\mathrm{O}$. Tsutsugamushi precludes generic immunity and to the allows reinjection. As it is a neglected disease, there is still a large gap in our knowledge of the disease, as evidence by the sporadic epidemiologic data and others relating to the public health information regarding scrub typhus in its endemic areas. It is a gram-negative bacterium, this infection can be perfectly treated with the specific antibiotics. Early treatment gives best results those who are infected with this infection it gives better therapeutic outcomes. Oral medication is effective for mild cases, but the parenteral route is often necessary for severely ill patients. Similar to treatment for other rickettsial diseases, doxycycline is one of most effective antibiotics for treating scrub typhus infection.
\end{abstract}

Key Words: Scrub typhus, threatens one billion peoples, gram-negative bacterium, Oral medication.

Article Info: Received; 03 Aug, 2018; Review Completed; 10 Sept, 2018; Accepted; 05 Oct, 2018; Available online: 20 Oct, 2018

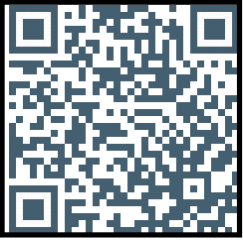

Cite this article as:

Nagaraju ,G.V., Kumar G.V.Pavan, Subramanyam Naga S.,An Extended Review and Summary on Facts about Etiology Epidemiology Clinical Diagnosis and Management of Scrub Typhus Infection, Asian Journal of Pharmaceutical research and Development. 2018; 6 (5):51-55 DOI: http://dx.doi.org/10.22270/ajprd.v6i5.404

*Address for Correspondence:

G.V.Nagaraju, Assistant Professor,Dept.of Pharmacy Practice,Koringa College of Pharmacy, Kakinada -533461, A.P, India

\section{INTRODUCTION}

$\mathrm{S}$ crub typhus or bus typhus inaction (Orientia tsutsugamushi infection) is transmitted by the bite of larval trombiculid mites and is endemic to the land mass within the triangle bounded by Japan to the north, Northern Australia to the south, and the Arabian Peninsula to the west. Mortality in the pre-antibiotic era was variable and in some series, approached $60 \%,{ }^{1}$ but specific and effective antimicrobial chemotherapy is now available.

Scrub typhus often presents as fever with little to distinguish it clinically from co-endemic diseases such as typhoid, leptospirosis, and dengue. The presence of an eschar supports the diagnosis but is variably present. $^{2}$ Diagnosis, therefore, depends on clinical suspicion, prompting the clinician to request an appropriate laboratory investigation, and failure to diagnose the disease will likely result in treatment with ineffective $\beta$-lactam-based regimens.
The mainstay in scrub-typhus diagnostics remains serology. The oldest test in current use is the Weil-Felix $\mathrm{OX}-\mathrm{K}$ agglutination reaction, which is inexpensive, easy to perform, and results are available overnight; however, it lacks specificity and sensitivity. The indirect fluorescent antibody (IFA) test is more sensitive, and results are available in a couple of hours; however, the test is more expensive and requires considerable training. IFA uses fluorescent anti-human antibody to detect specific antibody from patient serum bound to a smear of scrub-typhus antigen and is currently the reference standard.Indirect immunoperoxidase (IIP) eliminates the expense of a fluorescent microscope by substituting peroxidase for fluorescein ${ }^{3}$.

\section{Etiology and Epidemiology}

Scrub typhus is a life-threatening infectious disease that presents as an acute undifferentiated febrile illness. Its agent, Orientia tsutsugamushi, is an obligately intracytosolic bacterium that is transmitted by feeding larval trombiculid mites, which are the reservoir of the 
agent and the only life stage that feeds on a vertebrate host. Nymphal and adult trombiculid mites live in the soil and feed on the eggs of insects. Mites maintain the organisms by transovarian transmission as well as transtadial transmission through the mite's lifecycle ${ }^{3}$ Although mites transmit $O$. tsutsugamushi to vertebrate hosts such as rodents, only a small proportion of uninfected mites acquire Orientia during feeding on infected animals, and the ingested Orientia do not establish disseminated infection in the mites and are not transmitted transovarially to the next generation. ${ }^{4}$ It seems that chigger cofeeding on rodents is more relevant for effective mouse-to-mite transmission of Orientia than feeding on rickettsemic hosts. ${ }^{5}$ Thus, the true role of rodents as reservoirs requires additional investigation, because there may be marked genetic variability in chiggers with respect to the ability to acquire rickettsiae by feeding. Also, rodents may be better considered as dead-end hosts just as humans are rather than a reservoir. However, the evolutionary selection of tremendous antigenic diversity of the immunodominant major $56 \mathrm{kDa}$ surface protein presents an enigma if immune variation plays no role in survival of $O$. tsutsugamushi. ${ }^{6}$ The potential immunomodulatory effects of the saliva of larval mites on the pathogenesis of and immunity to infection with $O$. tsutsugamushi remain undetermined.

One million cases of scrub typhus occur each year with an estimated $10 \%$ case fatality rate unless treated appropriately, very likely resulting in more deaths than dengue. ${ }^{7}$ Currently, scrub typhus has predominantly been reported from an area extending from the Russian Far East and Korea in the north to northern Australia in the south and Afghanistan in the west, and it includes islands of the western Pacific and Indian Oceans, including Japan, Taiwan, Philippines, Papua New Guinea, Indonesia, and Sri Lanka. ${ }^{8}$ This geographical range may be an underrepresentation, because case reports have been published from Africa and South America. The recent isolation of a novel species $O$. chuto acquired by a patient in Dubai, the detection of another divergent Orientia transmitted to a patient in Chile, and serologic diagnoses of scrub typhus acquired in Africa indicate that a wider geographic distribution and genetic diversity of the genus should be investigated. The burden of disease in rural areas of Asia is large, with studies showing scrub typhus causing up to $20 \%$ of febrile hospital admissions, an incidence of infections of greater than $3 \%$ of the population monthly, seroprevalence over $50 \%$ of the population, despite a significant annual rate of reversion to seronegativity of $50 \%$ of cases, and a seroconversion rate of 484 per 1,000 person-years ${ }^{9}$.

In 1999, the World Health Organization (WHO) stated, "Scrub typhus is probably one of the most under diagnosed and underreported febrile illnesses requiring hospitalization in the region. This opinion remains valid today and could justifiably be adjusted to scrub typhus is probably the single most prevalent, under-recognized, neglected, and severe but easily treatable disease in the world. This ancient disease is currently undergoing increased awareness both because of re-emergence and rising incidence in previously unrecognized areas and improved diagnostic testing. It is apparent that scrub typhus has been recognized to occur frequently now in places where the illness was nearly forgotten, including
India, Sri Lanka, the Maldives, and Micronesia. However, scrub typhus has also emerged in regions north of the Yangtse River in China, where it was not known previously. The emergence of scrub typhus should also take into consideration the expansion of farmlands that have generated ideal habitats for trombiculid mites. The potential relationship with global climate change is unclear. Greater recognition in some countries, such as Thailand, Laos, Taiwan, and Japan, may reflect increased medical investigations and application of new diagnostic methods ${ }^{9}$. The critical unresolved issues regarding epidemiology emphasize the need to determine the reasons for emergence and re-emergence and the true incidence of this neglected disease, for which calculated days of disability-adjusted years of life lost (DALYs) have not been determined and to which appropriate attention has not been paid.

The traditional endemic area of scrub typhus is known as the tsutsugamushi triangle. It is a

Region covering more than 8 million $\mathrm{km} 2$, from the Russian Far East in the north, to Pakistan

in the west, Australia in the south, and the Japan in the east . There are one billion people at risk of infection; the endemic area is highly populated. The progress of globalization and associated travel contributes to the exportation of the infected persons to non endemic areas the antigenic and genetic diversity of $O$. tsutsugamushi strains, and their unclear correlation with virulence for humans, confounds the epidemiological study of scrub typhus ${ }^{10}$. Better understanding of the epidemiology of scrub typhus will help efforts to prevent and control the disease. This part of the article describes studies of the geographic distribution and risk factors of scrub typhus in both the endemic areas and in travellers from the rest of the world.

\section{Clinical Diagnosis}

The mainstay in scrub-typhus diagnostics remains serology. The oldest test in current use is the Weil-Felix OX-K agglutination reaction, which is inexpensive, easy to perform, and results are available overnight; however, it lacks specificity and sensitivity 3 The indirect fluorescent antibody (IFA) test is more sensitive, and results are available in a couple of hours; however, the test is more expensive and requires considerable training. IFA uses fluorescent anti-human antibody to detect specific antibody from patient serum bound to a smear of scrub-typhus antigen and is currently the reference tandard.4 Indirect immunoperoxidase (IIP) eliminates the expense of a fluorescent microscope by substituting peroxidise for fluorescein. 3 All currently available serological tests for scrub typhus have limitations in which clinicians need to be aware, despite their widespread use. Although agreement exists that $a \geq 4$ fold increase in antibody titer between two consecutive samples is diagnostic, 4 such a diagnosis is retrospective and cannot guide initial treatment. Diagnosis based on a single acute-serum sample requires using a cut-off antibody titer. Cut offs ranging from 1:10 to 1:400 are quoted, often with little corroborating evidence 4 and without establishing titers in the healthy local population (necessary to distinguish background immunity from acute infection); that cut off is then used for all patients, 
irrespective of PCR targeting the $56-\mathrm{kDa}$ antigen has been shown to be highly specific, 2,14 but sequence variability may affect primer annealing and therefore, test sensitivity. 15 A whole-blood-based assay targeting the 16S rRNA gene showed a sensitivity of only $37.5-52.3 \%$ (95\% confidence interval) in real-world conditions 15 , 16 (probably because median copy number was only 13 copies/ $\mathrm{mL}$ of blood 16 ), but it performed better than the $56-\mathrm{kDa}$ gene target in the same study (sensitivity $=22.5$ $36.1 \%$ ); however,this may have been caused by differences in the assay rather than differences in the target genes. 15 The $47-\mathrm{kDa}$ outer-membrane protein is highly specific for $\mathrm{O}$. tsutsugamushi , 17 and speciesspecific primers also exist for the highly conserved molecular chaperone gene, groEL . 13, 18 But, it remains to be seen if either target will allay concerns about detecting infection caused by previously undescribed serotypes. Loop isothermal amplification (LAMP) is a technique for amplifying DNA that makes use of three specially designed whether or not they come from a scrub-typhus-endemic environment. Although IFA may be modified to report separate IgG and IgM titers, there is no consensus on when this is useful or how to interpret the results. Currently available rapid bedside tests are based on serological methods and share the same inherent problems as IFA. Most frequently, IFA uses antigen from just three serotypes: Karp, Kato, and Gilliam. Yet, enormous antigenic variation has been found everywhere where it has been sought. 5 Eight different serotypes were found in mites from a single field in Malaysia. 6 In South Korea, $>75 \%$ of isolates are of the Boryong serotype. 7 On the Japanese island of Kyushu, $>90 \%$ of the disease reported is of the Kawasaki or Kuroki serotypes. 8 The Infectious Disease Surveillance Centre in Japan, therefore, recommends a two-pronged approach to diagnosis. First, local strains are included in the IFA for each prefecture; second, PCR of the blood clot is performed on all specimens, 8 although Buffy coat might be preferable. This recommendation is not widely implemented outside of Japan. 4 Isolating $\mathrm{O}$. tsutsugamushi requires bio safety level-3 facilities and culture on cell monolayers; median time to positivity is 27 days. 9 Mouse inoculations is even more laborious and intensive on resources. 10 Current methods of isolation are, therefore, not appropriate for the routine diagnosis of scrub typhus. There is an urgent need for alternative diagnostic methods; however, evaluation is hampered, because the current gold standard (IFA) is imperfect. In a Korean polymerase chain reaction (PCR) study of eschars, O. tsutsugamushi DNA was detected in six of seven patients who tested negative for scrub typhus by IFA but had eschars typical of scrub typhus. 11 In a study from Thailand, 3 of 20 (15\%) patients with fever had positive $O$. tsutsugamushi PCR, despite negative serology. 12 One recent study attempted to surmount this problem by evaluating the proposed test against a panel of serological and PCR-based methods, 13 but PCR is itself beset with problems. The high resource costs and training required make it impractical for many areas where scrub typhus is endemic. That aside, the most appropriate specimen to use remains unclear. PCR of eschar material is more sensitive than blood and remains positive even after the initiation of treatment. 11 Unfortunately, in a setting where eschars are present in
$7 \%$ of patients, 2 for example, eschar-based tests can have a maximum sensitivity of only $7 \%$. Using buffy coat could improve sensitivity compared with whole blood, 13 but bloodbased assays are positive only during the time window of rickettsemia. It the optimal PCR target remains unclear; nested primer pairs and the Bst DNA polymerase. There is no complicated DNA extraction procedure, and unlike PCR, the entire reaction takes place at the same temperature. This means that only a water bath or heating block is required, whereas PCR requires a thermocycler. The reaction is read visually (a positive reaction produces a white pellet) and does not require special equipment. 13 A small proof-of-principle study (nine patients) showed that LAMP could detect DNA levels as low as 14 copies/ $\mu \mathrm{L}$ compared with 3 copies/ $\mu \mathrm{L}$ for real-time PCR). 13 However, the technique has yet to be validated in a prospective clinical trial. Clinicians will remain dependent on serological methods until these issues are resolved, but work can be done to optimize their performance. Cut offs must be validated locally, and previously undiscovered serotypes must be assiduously searched for by examining rodents and chiggers, not merely BSL3 = bio safety level 3; + = low/poor; +++++ = high/excellent on a five-point qualitative scale. Validated against IFA alone but instead, be compared against a panel of both serological and antigen-detection assays (e.g., IFA with 47-kDA and/or 56-kDa PCR).

\section{Management Supportive Treatment}

Patients with mild disease presenting with fever without organ dysfunction may require only antipyretics along with antibiotics. Patients presenting with organ dysfunction would need organ support depending on the nature and extent of organ dysfunction ${ }^{10}$. Patients with respiratory failure could be supported either by means of non-invasive or invasive mechanical ventilation based on standard criteria in the management of respiratory failure. Those with circulatory shock can be treated with fluid resuscitation and vasoactive therapy if the blood pressure does not improve with fluids. Acute kidney injury, which is not uncommon in scrub typhus, may need renal replacement therapy. Those with DIC with clinical bleeding would require transfusion of blood products depending on the nature of coagulation derangement.

\section{Specific treatment}

The drug treatment options in scrub typhus have been evaluated and summarized in a recent meta-analysis. In the 17 studies that were included in the meta-analyses, six antibiotics were used and included doxycycline, chloramphenicol, azithromycin, rifampicin, roxithromycin and tetracycline. Conventionally, the treatment of scrub typhus involves the use of the tetracycline group of antibiotics or chloramphenicol. Since these drugs are contraindicated in pregnancy and in children, alternative agents such as quinolones and macrolides are used for the treatment of scrub typhus in this setting ${ }^{11}$.

In the four studies that compared azithromycin with chloramphenicol, chloramphenicol treatment was associated with significantly shorter median time to clearance of fever and lower adverse events when 
compared with azithromycin ${ }^{12}$. Six studies compared doxycycline with chloramphenicol; symptom clearance time was significantly shorter with doxycycline. No significant differences were observed in symptom duration comparing azithromycin with doxycycline (3 studies), roxithromycin with doxycycline (3 studies) and doxycycline with either rifampicin or tetracycline (2 studies each) ${ }^{13}$.

Doxycycline is the preferred drug in the treatment of scrub typhus. A therapeutic response to doxycycline therapy is used as a diagnostic test. In less sick patients oral doxycycline can be administered at $100 \mathrm{mg}$ twice daily. The duration of treatment is $7 \mathrm{~d}$. In critically ill patients, particularly those in shock, the absorption of enterally administered doxycycline may be problematic. In such situations, intravenous doxycycline should be used; where unavailable, intravenous azithromycin may be used in isolation or combined with enteral doxycycline. Azithromycin is also the recommended drug for treatment of scrub typhus in pregnancy. Rifampicin may be considered where doxycycline resistance is present. In one trial of patients with mild scrub typhus, Rifampicin was found to have shorter defervescence time when compared with doxycycline. However, in tuberculosis endemic countries, rifampicin should be avoided for the treatment of scrub typhus. Although there is some evidence for the use of quinolones in scrub typhus, recent reports of quinolone resistance suggests that this treatment should not be used in critically ill patients. Preliminary reports suggest that Telithromycin is a promising new antibacterial agent for patients with mild to moderate scrub typhus ${ }^{14}$. The different antimicrobial agents used in scrub typhus

\section{Current Prevention Methods}

There is no vaccine available for any rickettsial infections including scrub typhus. The enormous antigenic variation in different $\mathrm{O}$. Tsutsugamushi strains, and weak and short lived cross protection among different strains hamper the development of an effective vaccine. Vaccine efforts are also hindered by the different antigenically divergent strains of $O$. tsutsugamushi in different endemic countries/regions, or even among different strains in the same location WHO recommends prophylactic treatment under special circumstances in the endemic areas. A single oral dose of doxycycline, chloramphenicol or tetracycline every 5 days for a total of 35 days provided protection against Orientia infection. A prospective randomized double blind study among Taiwanese military personnel confirmed that prophylactic treatment with doxycycline could decrease the incidence of scrub typhus to $1 / 5$ of that in the placebo group ${ }^{15}$. The US army has used a weekly dose of doxycycline to prevent scrub typhus. However, CDC in the U.S. does not recommend using antibiotics as prophylaxis for rickettsial diseases including scrub typhus because the preventive treatment may simply delay onset of disease and make diagnosis more difficult. To treat rickettsial diseases more effectively, CDC suggests starting treatment based on clinical suspicion alone ${ }^{16}$.

General protective measures can be followed to avoid the infection. These precautions are critical for people living in or visiting endemic areas
Avoid outbreaks. Persons should avoid known focal outbreak areas to the extent that this is Possible. Travellers can check the regional disease transmission and outbreak information at http://www.cdc.gov/travel.

Avoid exposure conditions. Chiggers reside in grass, woodlands, and other vegetated areas. Persons are encouraged to avoid the outdoors or take preventive actions. Do not sit or lie on bare ground or grass; use a sheet or a cover on the ground instead.

1. Wear appropriate clothing. Persons should wear longsleeved shirts, long pants, boots and

2. Hats to reduce exposure. Persons should tuck in shirts and pants, and wear closed shoes.

3. Insect and spatial repellents. Persons should apply insect repellent containing dibutyl Phthalate, benzyl benzoate, diethyl toluamide or other chemicals to their skin and permethrin to their clothing, to prevent chigger bites.

4. Insecticides and habitat modification. Farmers and field workers can improve sanitation, Clear vegetation, control rodents, use insecticides and chemically treat the soil. These steps can impede the propagation of chiggers and the transmission cycle.

5. Thorough cleaning after visiting high risk areas. Due to its small size of $0.2 \pm 0.4 \mathrm{~mm}$, detection of mite larva on clothing or skin is extremely difficult. Prompt removal of clothing and thorough cleaning of skin and clothes with detergent after work or travel and at the end of the day can reduce the risk of infection.

\section{CONCLUSION}

I concluded that the scrub typhus infection can be caused by the Orientia tsutsugamushi, is an obligately intracytosolic bacterium that is transmitted by feeding larval trombiculid mites and it can be treated with the specific antibiotics. Early treatment gives best results those who are infected with this infection it gives better therapeutic outcomes. Oral medication is effective for mild cases, but the parenteral route is often necessary for severely ill patients. Similar to treatment for other rickettsial diseases, doxycycline is one of most effective antibiotics for treating scrub typhus infection.WHO propose or the prophylactic treatment under special circumstances in the endemic areas and it can be used a single oral dose of doxycycline, chloramphenicol or tetracycline every 5 days for a total of 35 days provided protection against Orientia infection.

\section{REFERENCES}

1. Kelly DJ, Fuerst PA, Ching WM, Richards AL (2009) Scrub typhus: the geographic distribution of phenotypic and genotypic variants of Orientia tsutsugamushi. Clin Infect Dis 48 Suppl 3: S203 \pm 230

2. Valbuena G, Walker DH (2013) Approaches to vaccines against Orientia tsutsugamushi. Frontiers in Cellular and Infection Microbiology 2.

3. Kim I-S, Walker DH (2011) Scrub Typhus. In: Guerrant RL, Walker DH, Weller PF, editors. Tropical infectious diseases: principles, pathogens and practice. Third ed: Elsevier Health Sciences. pp. 332 \pm 336 .

4. Paris DH, Shelite TR, Day NP, Walker DH (2013) unresolved problems related to scrub typhus: A seriously neglected lifethreatening disease. American Journal of Tropical Medicine and Hygiene 89: 301 \pm 307 . https://doi.org/10.4269/ajtmh.13-0064 PMID: 23926142 
5. Warrell DA, Cox TM, Firth JD (2003) Oxford textbook of medicine: Oxford University Press.

6. Mahajan SK (2005) Scrub typhus. J Assoc Physicians India 53: 954 \pm 958 . PMID: 16515236

7. Jeong YJ, Kim S, Wook YD, Lee JW, Kim KI, et al. Scrub typhus: clinical, pathologic, and imaging findings. Radiographics. 2007/01/20 ed. pp.

8. Tsay RW, Chang FY (1998) Serious complications in scrub typhus. J Microbiol Immunol Infect 31:240 244 . PMID: 10496165

9. Jamil M, Lyngrah KG, Lyngdoh M, HussainM, Clinical manifestations and complications ofscrub typhus: a hospital based study from north eastern India. J Assoc Physicians India, 2014;62: 19 \pm 23 .

10. Groves MG, Harrington KS, Scrub typhus. Beran GW, ed.Handbook of Zoonoses. Section A: Bacterial, Rickettsial,Chlamydial, and Mycotic Zoonoses . 2nd ed . Boca Raton, FL: CRC Press, 1994; 463 - 474.

11. Silpapojakul K, Varachit B , Silpapojakul K, Paediatric scrub typhus in Thailand: a study of 73 confirmed cases . Trans R Soc Trop Med Hyg, 2004; 98: 354 - 359.
12. Kelly DJ, Wong PW, Gan E, Lewis GE, Comparative evaluation of the indirect immunoperoxidase test for the serodiagnosis of rickettsial disease. Am J Trop Med Hyg, 1988; 38: 400 - 406.

13. Blacksell SD, Bryant NJ , Paris DH, Doust JA, Sakoda Y, Day NPJ , Scrub typhus serologic testing with the indirect immunofluorescence method as a diagnostic gold standard: a lack ofbconsensus leads to a lot of confusion . Clin Infect Dis, 2007. 44: $391-401$.

14. Traub R, Wisseman CL Jr, The ecology of chigger-borne rickettsiosis (scrub typhus). J Med Entomol,1974; 11: 237-303.

15. Roberts LW, Rapmund G, Cadigan FC Jr, Sex ratios in Rickettsia tsutsugamushi-infected and noninfected colonies of Leptotrombidium (Acari:Trombiculidae). J Med Entomol1977; 14: 89-92.

16. Roberts LW, Muul I, Robinson DM, Numbers of Leptotrombidium (Leptotrombidium) deliense (Acarina: trombiculidae) and prevalence of Rickettsia tsutsugamushi in adjacent habitats of peninsular Malaysia. Southeast Asian J Trop Med Public Health 1977; 8: 207-213. 